\title{
REFLEXÃO ACERCA DA INTERFACE ENTRE A SEGURANÇA DO PACIENTE E
} O PROCESSO DE ENFERMAGEM

\section{REFLECTION ON THE INTERFACE BETWEEN PATIENT SAFETY AND THE NURSING PROCESS}

\section{REFLECTION ACERCA DE LA INTERFAZ ENTRE LA SEGURIDAD PACIENTE Y EL PROCESO DE ENFERMERÍA}

\author{
Edlamar Kátia Adamy ${ }^{1}$, Fernanda Karla Metelski ${ }^{2}$, Carla Argenta ${ }^{3}$, Olvani Martins da Silva ${ }^{4}$ \\ Denise Antunes de Azambuja Zocche
}

\section{RESUMO}

Objetivo: Este estudo busca apresentar uma reflexão acerca das relações entre a segurança do paciente e o processo de enfermagem, com vistas a estimular o debate sobre o tema nos espaços que oferecem saúde e formação profissional. Método: Percebe-se que, em interface com a segurança do paciente, o Processo de Enfermagem destaca-se como aliado a assistência, uma vez que, padronizando as ações de enfermagem, está-se contribuindo para o alcance das metas de segurança. Resultados: Ações como incorporação de novos hábitos relacionados às práticas de segurança, gestão de risco, produção e validação de protocolos construídos a partir das etapas do Processo de Enfermagem estabelecem a conexão de garantia de segurança do paciente. Conclusão: O Processo de Enfermagem, por si só, constitui um dispositivo transversal às metas de segurança do paciente e está diretamente relacionado à qualificação da assistência de enfermagem.

Descritores: Enfermagem; Processo de enfermagem; Segurança do paciente.

\footnotetext{
${ }^{1}$ Enfermeira. Doutora em Enfermagem pela Universidade Federal do Rio Grande do Sul. Professora Adjunta do Departamento de Enfermagem e do Programa de Pós Gradução em Enfermagem da UDESC.

${ }^{2}$ Enfermeira, Mestre em Políticas Sociais e Dinâmicas Regionais pela Unochapecó, Professora Assistente da UDESC.

3 Enfermeira, Doutoranda em Enfermagem pela Universidade Federal do Rio Grande do Sul- UFRGS, Professora Assistente da UDESC.

${ }^{4}$ Enfermeira. Doutora em Enfermagem pela Universidade Federal do Rio Grande do Sul. Professora Adjunta do Departamento de Enfermagem.

${ }^{5}$ Enfermeira. Doutora em Enfermagem pela Universidade Federal do Rio Grande do Sul. Professora Adjunta do Departamento de Enfermagem e do Programa de Pós Graduação em Enfermagem da UDESC.
} 


\begin{abstract}
Objective: This study aims to present a reflection on the relationship between patient safety and the nursing process with a view to stimulating the debate on the subject in the spaces where health is produced and in professional training. Method: It is noticed that in interface with patient safety the Nursing Process stands out as allied to care, since, by standardizing the nursing actions, we will be contributing to the achievement of safety goals. Results: Through the incorporation of new habits related to safety practices, risk management, production and validation of protocols constructed from the steps of the Nursing Process, the connection of patient safety assurance is established. Conclusion: The Nursing Process, by itself, is a device that is transversal to the patient's safety goals and is directly related to the qualification of nursing care.
\end{abstract}

Descriptors: Nursing; Nursing process; Patient safety.

\title{
RESUMEN
}

Objetivo: Este estudio tiene como objetivo presentar una reflexión acerca de las relaciones entre la seguridad del paciente y el proceso de enfermería, con vistas a estimular el debate sobre el tema en los espacios que ofrecen salud y formación profesional. Metodología: Se percibe que, en interfaz con la seguridad del paciente, el Proceso de Enfermería se destaca como aliado a la asistencia, una vez que, estandarizando las acciones de enfermería, se está contribuyendo al logro de las metas de seguridad. Resultados: Las acciones como incorporación de nuevos hábitos relacionados a las prácticas de seguridad, gestión de riesgos, producción y validación de protocolos construidos a partir de las etapas del Proceso de Enfermería para establecer una conexión de seguridad del paciente. Conclusión: El proceso de enfermería, por sí solo, constituye un dispositivo transversal a las metas de seguridad del paciente y está directamente relacionado a la calificación de la asistencia de enfermería.

Descriptores: Enfermería; Proceso de Enfermería; Seguridad del paciente.

\section{INTRODUÇÃO}

A temática segurança do paciente tem sido foco de debate das principais organizações de saúde no mundo, principalmente nas duas últimas décadas. Em 2008, a Organização Pan-Americana da Saúde (OPAS) criou a Rede Internacional de Enfermagem e Segurança do Paciente (RIENSP), com o objetivo de trocar informações e fortalecer o cuidado de enfermagem por meio de evidências científicas. $^{1}$ No Brasil, a rede foi consolidada com a criação da Rede Brasileira de Enfermagem e Segurança do Paciente (REBRAENSP), cuja meta principal é articular as instituições de saúde e educação, visando fortalecer a assistência de enfermagem segura e com qualidade. $^{2}$

O Programa Nacional de Segurança do Paciente (PNSP) foi instituído em 2013, 
e tem por objetivo contribuir para a qualificação do cuidado em saúde em todos os estabelecimentos de saúde do território nacional. ${ }^{3}$ A preocupação com a segurança do paciente, contudo, antecede o programa e vem sendo difundida pela Agência Nacional de Vigilância Sanitária (ANVISA) desde a publicação dos requisitos de boas práticas de funcionamento dos serviços de saúde e pela obrigatoriedade de implantação dos Núcleos de Segurança do Paciente (NSP). ${ }^{4}$

Os NSP vêm sendo implantados a fim de promover ações que visem à melhoria da segurança do paciente e a qualidade do cuidado em saúde, por meio de um processo de construção consensual entre os diversos atores que dele participam. ${ }^{3}$ Nesse sentido, destaca-se que a segurança é um princípio básico e um requisito para a qualidade do cuidado, que, por sua vez, é uma exigência nos setores de saúde, por meio da gestão do processo de trabalho coletivo. ${ }^{5}$

Para os profissionais da enfermagem, está posto o desafio de atender às diretrizes do PNSP, incorporando esses conceitos na cultura institucional, por meio das atividades desenvolvidas no cotidiano do trabalho em saúde e enfermagem. Parte-se do principio de que a enfermagem é a ciência do cuidado, e todo cuidado deve ser implementado com segurança. Nesse sentido, as atividades privativas do enfermeiro asseguram práticas de atenção à saúde de forma sistematizada, a exemplo do processo de enfermagem (PE), que, além de conferir visibilidade profissional para a categoria, busca qualificar a assistência ao usuário, momento da produção do cuidado.

É por meio do PE que as ações dos enfermeiros tornam-se elementos fundamentais para consolidar uma prática de enfermagem científica, uma vez pautada nos preceitos da segurança e da qualidade do cuidado. $^{6}$ Dessa forma, o PE se apresenta como um marco legal da profissão que orienta para o registro e a organização do cuidado, garantindo a documentação da prática profissional, e, consequentemente, a segurança do paciente.

Diante de tais prerrogativas, este estudo tem como objetivo apresentar uma reflexão acerca das relações entre a segurança do paciente e o processo de enfermagem, com vistas a estimular $\mathrm{o}$ debate sobre o tema nos espaços que produzem saúde e na formação profissional. 


\section{A SEguranÇA dO PACIENTE E SUA RELAÇÃO COM O PROCESSO DE ENFERMAGEM}

Conceitualmente, a segurança pode ser definida como a redução do risco de danos desnecessários, associados à atenção à saúde, até um mínimo aceitável. ${ }^{1,4} \mathrm{~A}$ segurança do paciente está diretamente ligada à minimização dos eventos adversos, ou seja, danos evitáveis (erros por negligência) e inevitáveis (como o efeito colateral de um medicamento, por exemplo). ${ }^{7}$

Os possíveis eventos adversos, decorrentes de práticas inseguras, precisam ser trabalhados com as equipes de enfermagem e problematizados em meio ao trabalho em ato, ou mesmo em rodas de conversa, para que todos tenham sua compreensão, e saibam identificar quando ocorre um dano evitável, ou mesmo um inevitável, a diferença entre eles, a forma de agir nas diferentes situações, e a importância de realizar a notificação.

Nesse contexto, o PE pode ser considerado um importante fator para identificar, prevenir e minimizar os eventos adversos nos diferentes contextos da prática profissional, uma vez que busca organizar uma assistência baseada em evidências, fundamentada cientificamente, respaldada legalmente, e documentada, o que contribui para a segurança do paciente e do profissional. Corroborando, a ANVISA menciona que a segurança do paciente envolve a qualificação do cuidado em saúde com o foco em pacientes, profissionais de saúde e ambientes de assistência à saúde. ${ }^{4}$

As evidências advindas da implementação do PE, quando registradas de maneira completa e fidedigna, podem assegurar a continuidade da assistência e promover a segurança do paciente. $^{8}$ Registrar no prontuário do paciente as informações inerentes e indispensáveis ao processo de cuidar é dever profissional, conforme previsto no Código de Ética de Enfermagem e nas Resoluções COFEN$358 / 2009^{9}$ e 429/2012. ${ }^{10}$ No momento em que o enfermeiro registra informações do paciente, da família ou da comunidade, fomenta uma reflexão crítica sobre os problemas evidenciados e dos resultados esperados para, então, definir as intervenções de enfermagem e os cuidados que serão prestados.

Assim, em interface com a segurança do paciente, destaca-se que o PE é um aliado para a assistência, uma vez que, padronizando as ações de enfermagem 
por meio da identificação de problemas (diagnósticos) e riscos individuais e coletivos, bem como com a avaliação das intervenções de enfermagem a partir dos resultados, contribui-se para o alcance das metas de segurança do paciente, fortalecendo o cuidado de enfermagem por meio de evidências científicas.

O PE corrobora as normativas legais acerca da temática segurança do paciente no que tange ao cumprimento das metas básicas, prevista pelo PNSP, considerando: identificação correta do paciente; a comunicação entre profissionais de saúde; a segurança na prescrição, no uso e na administração de medicamentos; a realização de cirurgia segura em sítio cirúrgico, procedimento e pacientes corretos; a higienização das mãos para evitar infecções e a avaliação do paciente, em relação ao risco de quedas, úlcera por pressão, estabelecendo ações preventivas. ${ }^{1}$ Essa relação é plausível por meio da implantação de protocolos básicos que contemplem o desenvolvimento do PE a fim de legitimar a qualidade do cuidado ofertado.

É possível fomentar a segurança do paciente em interface com o PE por meio da incorporação de novos hábitos relacionados às práticas de segurança e gestão de risco, a produção e validação de protocolos, guias e manuais voltados à segurança do paciente em diferentes contextos, construídos a partir das etapas do PE e utilizando-se do sistema de linguagem padronizada que oferecem uma estrutura para organizar os diagnósticos, intervenções e resultados de enfermagem.

\section{CONSIDERAÇÕES FINAIS}

A interface entre a segurança do paciente e o PE precisa ser mais explicitada, uma vez que agrega e fortalece o cuidado de enfermagem e a prática profissional. O PE, por si só, constitui um dispositivo transversal às metas de segurança do paciente e está diretamente relacionado à qualificação da assistência de enfermagem nos procedimentos e na aplicação dos protocolos que envolvem a produção do cuidado.

Cabe ressaltar que as gerências de enfermagem são responsáveis por fomentar uma visão e uma missão institucional, como suporte para que os enfermeiros tenham condições de aplicar o PE em todas as suas fases, contemplando e assegurando as metas preconizadas pelo Programa Nacional de Segurança do Paciente, além dos padrões preconizados pelas agências certificadoras. 


\section{REFERÊNCIAS}

1. Ministério da Saúde (Brasil); Fundação Oswaldo Cruz; Agência Nacional de Vigilância Sanitária. Documento de referência para o Programa Nacional de Segurança do Paciente. Brasília, DF: Ministério da Saúde; 2014 [citado em 10 fev 2016]. Disponível em: http://bvsms.saude.gov.br/bvs/publi cacoes/documento_referencia_prog rama_nacional_seguranca.pdf

2. Cassiani SHB. Enfermagem e a pesquisa sobre segurança dos pacientes [Editorial]. Acta Paul Enferm. [Internet] 2010 [citado em 18 fev 2016]; 23(6). Disponível: http://www.scielo.br/pdf/ape/v23n6 /01.pdf

3. Ministério da Saúde (Brasil). Portaria $n^{\circ} 529$, de $1^{\circ}$ de abril de 2013. Institui o Programa Nacional de Segurança do Paciente (PNSP) [Internet]. Brasília, DF: Ministério da Saúde; 2013 [citado em $21 \mathrm{dez}$ 2015]. Disponível: http://bvsms.saude.gov.br/bvs/saud elegis/gm/2013/prt0529_01_04_20 13.html

4. Agência Nacional de Vigilância Sanitária (Brasil). Implantação do Núcleo de Segurança do Paciente em Serviços de Saúde [Internet]. Brasília, DF: ANVISA; 2016 [citado em $10 \mathrm{dez} 2017$ ]. Disponível em: http://portal.anvisa.gov.br/documen ts/33852/3507912/Caderno+6++Implantação+do+Núcleo+de+Seg urança+do+Paciente+em+Serviços +de+Saúde/cb237a40-ffd1-401fb7fd-7371e495755c

5. Nogueira JWS, Rodrigues MCS. Comunicação efetiva no trabalho em equipe em saúde: desafio para a segurança do paciente. Cogitare Enferm [Internet] 2015 [citado em 01 fev 2016]; 20(3):636-40.

Disponível em: http://ojs.c3sl.ufpr.br/ojs2/index.ph $\mathrm{p} /$ cogitare/article/view/40016/2624 5

6. Menezes MFB, Souza CQ, Alcântara LFFL, Rodrigues MF, Vidal MLB. A interface da SAE com o processo de acreditação hospitalar: relato de experiência em uma instituição oncológica [Internet]. Rio de Janeiro: INCA; [2008] [citado em $20 \mathrm{dez} 2015$ ]. Disponível: http://bvsms.saude.gov.br/bvs/publi cacoes/inca/Fatima_Batalha_interfa ce_sae.pdf

7. Mendes W, Martins M, Rozenfeld $\mathrm{S}$, Travassos C. The assessment of adverse events in hospitals in Brazil. Int J Qual Health Care [Internet]. 2009 [citado em $01 \mathrm{fev}$ 2016]; 21(4):279-84. Disponível em:

https://academic.oup.com/intqhe/art icle/21/4/279/1801452

8. Lucena AF. Processo de enfermagem: interfaces com o processo de acreditação hospitalar [Editorial]. Rev Gaúch Enferm. [Internet]. 2013 [citado em $21 \mathrm{dez}$ 2015]; 34(4):8-9. Disponível em: http://seer.ufrgs.br/index.php/Revis taGauchadeEnfermagem/article/vie w/45306/28590

9. Conselho Federal de Enfermagem (Brasil). Resolução n. 358, de 15 outubro 2009. Dispõe sobre a sistematização da assistência de enfermagem e a implementação do Processo de Enfermagem em ambientes públicos ou privados, em que ocorre o cuidado profissional 
de Enfermagem, e dá outras providencias. Brasília, DF:

COFEN; 2009.

10. Conselho Federal de Enfermagem

(Brasil). Resolução n. 429, de 08 junho 2012. Dispõe sobre o registro das ações profissionais no prontuário do paciente, e em outros documentos próprios da enfermagem, independente do meio de suporte - tradicional ou eletrônico. Brasília, DF: COFEN; 2012.

RECEBIDO: $16 / 01 / 2018$ APROVADO: $26 / 06 / 2018$ PUBLICADO: 07/2018 